\title{
The Habitability of GJ 357D: Possible Climate and Observability
}

\author{
L. Kaltenegger ${ }^{1,2}$, J. Madden ${ }^{1,2}$ (1) Z. Lin $^{1,2}$, S. Rugheimer ${ }^{1,3}$, A. Segura ${ }^{4}$, R. Luque ${ }^{5,6}$, E. Palle ${ }^{5,6}$, and N. Espinoza ${ }^{7}$ (1)

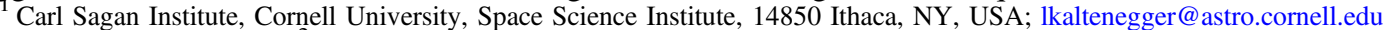 \\ ${ }^{2}$ Cornell University, Space Science Institute, 14850 Ithaca, NY, USA \\ Oxford University, Oxford OX1 3DW, UK \\ ${ }^{4}$ Instituto de Ciencias Nucleares, Universidad Nacional Autónoma de México, México \\ ${ }^{5}$ Instituto de Astrofisica de Canarias, E-38205 La Laguna, Tenerife, Spain \\ ${ }^{6}$ Departamento de Astrofisica, Universidad de La Laguna, E-38206, La Laguna, Tenerife, Spain \\ ${ }^{7}$ Max Planck Institute, Koenigstuhl 17, D-69117 Heidelberg, Germany \\ Received 2019 June 28; revised 2019 July 29; accepted 2019 July 29; published 2019 September 27
}

\begin{abstract}
The GJ 357 system harbors three planets orbiting a bright, nearby M2.5V star at $9.44 \mathrm{pc}$. The innermost planet, GJ 357b (TOI-562.01), is a hot transiting Earth-sized planet with Earth-like density, which receives about 12 times the irradiation Earth receives from the Sun, and was detected using data from TESS. Radial velocities discovered two more planets in the system at 9.12 (GJ $357 \mathrm{c}$ ) and 55.6 days (GJ $357 \mathrm{~d}$ ), with minimum masses of $3.59 \pm 0.50$ and $6.1 \pm 1$ Earth masses, and an irradiation of 4.4 and 0.38 Earth's irradiation, respectively. GJ $357 \mathrm{~d}$ receives slightly less stellar irradiation than Mars does in our own solar system, which puts it in the Habitable Zone (HZ) for its host star. GJ $357 \mathrm{~d}$ could not have been detected with TESS and whether it transits remains an open question. Here we model possible conditions within which GJ357 d could sustain surface habitability and present planetary models as well as synthetic transmission, reflection, and emission spectra for a range of models for GJ $357 \mathrm{~d}$ from water worlds to Earth-like models. With Earth-analog outgassing rates, GJ $357 \mathrm{~d}$ would be a frozen rocky world; however, with an increased $\mathrm{CO}_{2}$ level, as would be expected if a geological cycles regulates $\mathrm{CO}_{2}$ concentration like on Earth, the planet models show temperate surface conditions. If we can detect a transit of GJ $357 \mathrm{~d}$, it would become the closest transiting, potentially habitable planet in the solar neighborhood. Even if GJ $357 \mathrm{~d}$ does not transit, the brightness of its star makes this planet, in the $\mathrm{HZ}$ of a close-by M star, a prime target for observations with Extremely Large telescopes as well as future space missions.
\end{abstract}

Unified Astronomy Thesaurus concepts: Exoplanet atmospheres (487); Exoplanet astronomy (486); Habitable zone (696); Planet hosting stars (1242)

Supporting material: data behind figures

\section{Introduction}

To date more than 4000 exoplanets have been discovered, providing a first glimpse of the diversity of other worlds (e.g., reviews by Udry \& Santos 2007; Winn \& Fabrycky 2015). Several of these planets receive irradiation from their host star that is similar to Earth, which could provide liquid water and habitable surface environments for rocky planets or moons (see e.g., review Batalha 2014; Kane et al. 2016, Kaltenegger 2017).

For several hundred exoplanets both mass and radius are known and thus we can estimate the mean density of the planet, which can be used to derive its composition and compare it to planets in our own solar system. Figure 1 shows the diversity of small known exoplanets with the error bars on the measurements. We chose $4 R_{\text {Earth }}$ and $20 M_{\text {Earth }}$ as limits in Figure 1 to include the most massive known rocky planets, so-called Super-Earths like Kepler-10 c, a planet with about $18 M_{\text {Earth }}$ and 2.3 $R_{\text {Earth }}$ (Dumusque et al. 2012) consistent with a rocky composition (see Zeng \& Sasselov 2013). However, several known exoplanets with masses down to $1 M_{\text {Earth }}$, have radii corresponding to gas planets or so-called Mini-Neptunes; e.g., Kepler11-f has a mass between 1.1 and $5 M_{\text {Earth }}$, but a radius of 2.6 $R_{\text {Earth }}$ (Lissauer et al. 2011).

Colored lines in Figure 1 show exoplanetary density models for different composition from Iron $(100 \% \mathrm{Fe})$ to Earth-like ( $\mathrm{MgSiO} 3$ (rock)) to a pure $\mathrm{H}_{2} \mathrm{O}$ composition $\left(100 \% \mathrm{H}_{2} \mathrm{O}\right.$ ), encompassing the densest to lightest rocky composition for an exoplanet (following Zeng et al. 2016). Earth and Venus are shown in black for reference. Figure 1 shows that gas planets can have masses down to $1 M_{\text {Earth }}$, while planets with masses up to $18 M_{\text {Earth }}$ can also be rocky, making the mass of a planet a very weak constraint on its composition. The color-coding in Figure 1 indicates the effective surface temperature of the host star, which provides additional insight into the composition of small mass planets by host star spectral type.

GJ $357 \mathrm{~b}$ is the only planet in this system where a transit was detected so far, its radius is $1.217 \pm 0.084$ Earth radii, $R_{\text {Earth }}$,

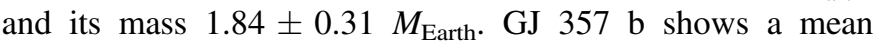
density like Earth, while both GJ 357 c and GJ 357 d currently only have minimum masses from radial velocity (RV) measurements, thus we do not know their radii or bulk composition. Figure 1 shows that in the region of minimum mass range for these two planets a wide variety of exoplanets from Super-Earths to Mini-Neptunes have been detected. The effective surface temperature of the host star indicates most planets in these mass ranges for $\mathrm{M}$ dwarfs, shown in red in Figure 1, are consistent with a rocky composition.

While a wide range of composition and atmosphere is possible for GJ $357 \mathrm{~d}$, we focus on the interesting case that this planet could have a rocky composition (see Figure 1). We model a range of scenarios for Gl $375 \mathrm{~d}$, from a planet model similar to Earth, which leads to a rocky radius of 1.75 (Zeng \& Sasselov 2013) and gravity of twice of Earth's to the limiting case for the largest rocky planet of this mass, a water world, 


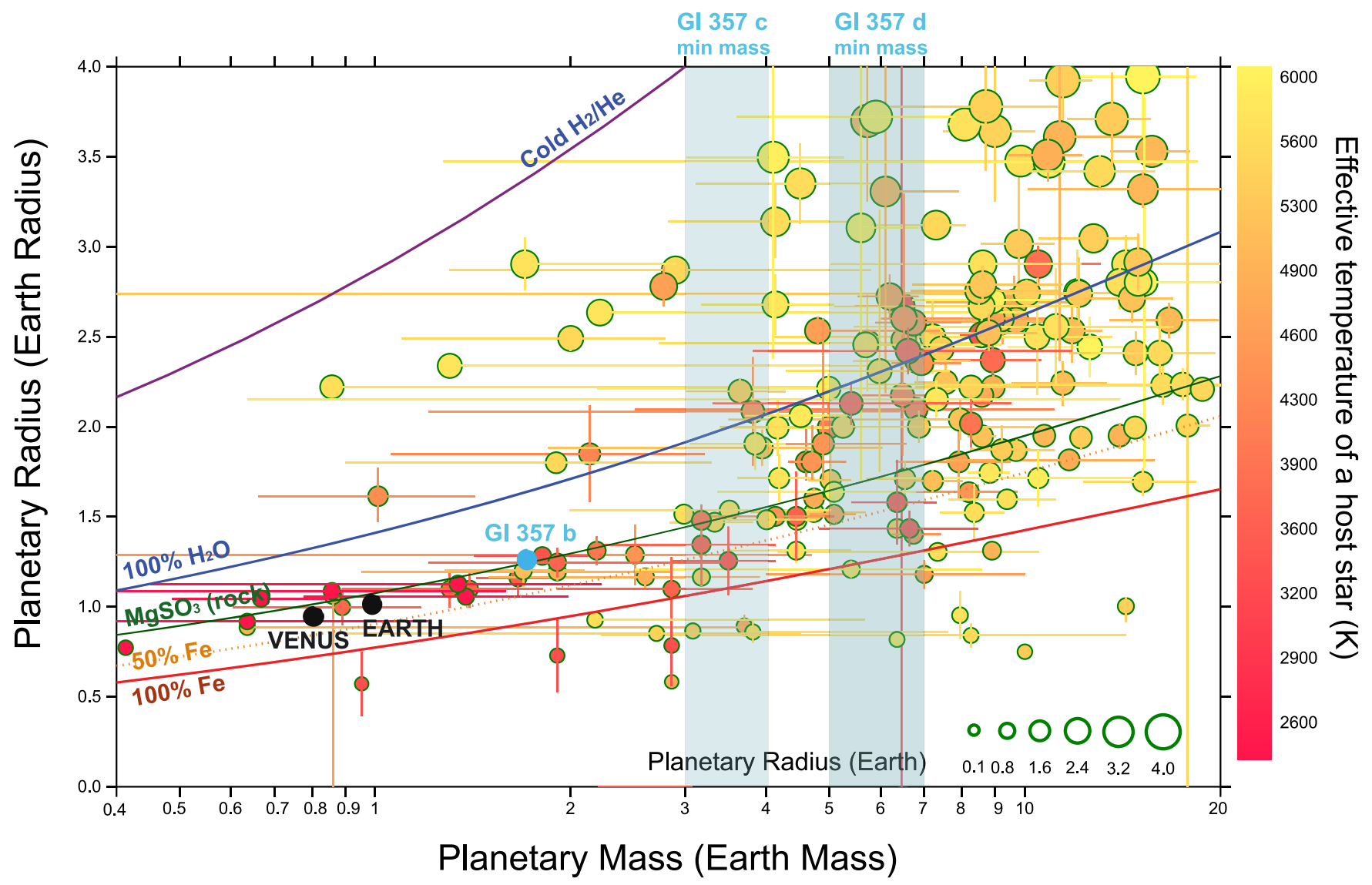

Figure 1. Mass radius diagram of detected exoplanets that have both mass and radius measurements (data exoplanet.eu, 2019 June 19).

which leads to a rocky radius of $2.4 R_{\text {Earth }}$ and a gravity of 1.16 times Earth's for Gl 375 d.

We can use the incident stellar flux planets receive to compare planetary environments: present-day Venus, for example, receives 1.9 times the Solar Flux at Earth's orbit, $S_{0}$, while present-day Mars receives $0.4 S_{\text {Earth }}$. Any rocky planet that receives more flux than present-day Venus is empirically too hot to be habitable. GJ 357 b and GJ $357 \mathrm{c}$ receive about 13 times and 4.4 times the Earth's irradiation $\left(S_{\text {Earth }}\right)$, respectively. For comparison, Venus receives about 1.9 $S_{\text {Earth }}$ and Mercury about $6.5 \mathrm{~S}_{\text {Earth }}$. Thus, both planets should have undergone a runaway greenhouse stage as proposed for Venus' evolution and lost their water. On the other hand, GJ $357 \mathrm{~d}$ receives an irradiation of $0.38 S_{\text {Earth }}$, which places it inside the Habitable Zone (HZ), in a location that is comparable to Mars in the solar system, making it a very interesting target for further atmospheric observations.

The $\mathrm{HZ}$ is a concept that is used to guide remote observation strategies to characterize potentially habitable worlds: it is defined as the region around one or multiple stars in which liquid water could be stable on a rocky planet's surface (e.g., Kasting et al. 1993; Kaltenegger \& Haghighipour 2013; Kane \& Hinkel 2013; Kopparapu et al. 2013; Ramirez \& Kaltenegger 2017), facilitating the remote detection of possible atmospheric biosignatures. The width and orbital distance of a given HZ depends to a first approximation on two main parameters: incident stellar flux and planetary atmospheric composition. The incident stellar flux depends on stellar luminosity, stellar spectral energy distribution (SED), the planet's orbital distance (semimajor axis), and eccentricity of the planetary orbit. The warming due to atmospheric composition depends on the planet's atmospheric makeup, energy distribution, and resulting albedo and greenhouse warming.

A star's radiation shifts to longer wavelengths with cooler surface temperatures, which makes the light of a cooler star more efficient at heating an Earth-like planet with a mostly $\mathrm{N}_{2}-\mathrm{H}_{2} \mathrm{O}-\mathrm{CO}_{2}$ atmosphere (see e.g., Kasting et al. 1993). This is partly due to the effectiveness of Rayleigh scattering, which decreases at longer wavelengths. A second effect is the increase in near-infrared (NIR) absorption by $\mathrm{H}_{2} \mathrm{O}$ and $\mathrm{CO}_{2}$ as the star's spectral peak shifts to these wavelengths, meaning that the same integrated stellar flux that hits the top of a planet's atmosphere from a cool red star warms a planet more efficiently than the same integrated flux from a star with a higher effective surface temperature (see Figure 2). Stellar luminosity, as well as the SED, change with stellar spectral type and age, which influences the orbital distance at which an Earth-like planet can maintain climate conditions that allow for liquid water on its surface (see a review by Kaltenegger 2017).

Figure 2 shows the empirical $H Z$, which is based on observations in our own solar system (see Kasting et al. 1993). The inner edge of this empirical HZ, the so-called Recent Venus limit, is based on the observation that Venus may have had liquid water on its surface until about 1 billion years ago, which is consistent with atmospheric $\mathrm{D} / \mathrm{H}$ ratio measurements suggesting a high initial water endowment (Donahue \& Pollack 1983). Note that the inner limit is not well known because of the lack of a reliable geological surface history of Venus beyond about 1 billion years due to resurfacing of the stagnant lid, which allows for the possibility of a liquid surface 

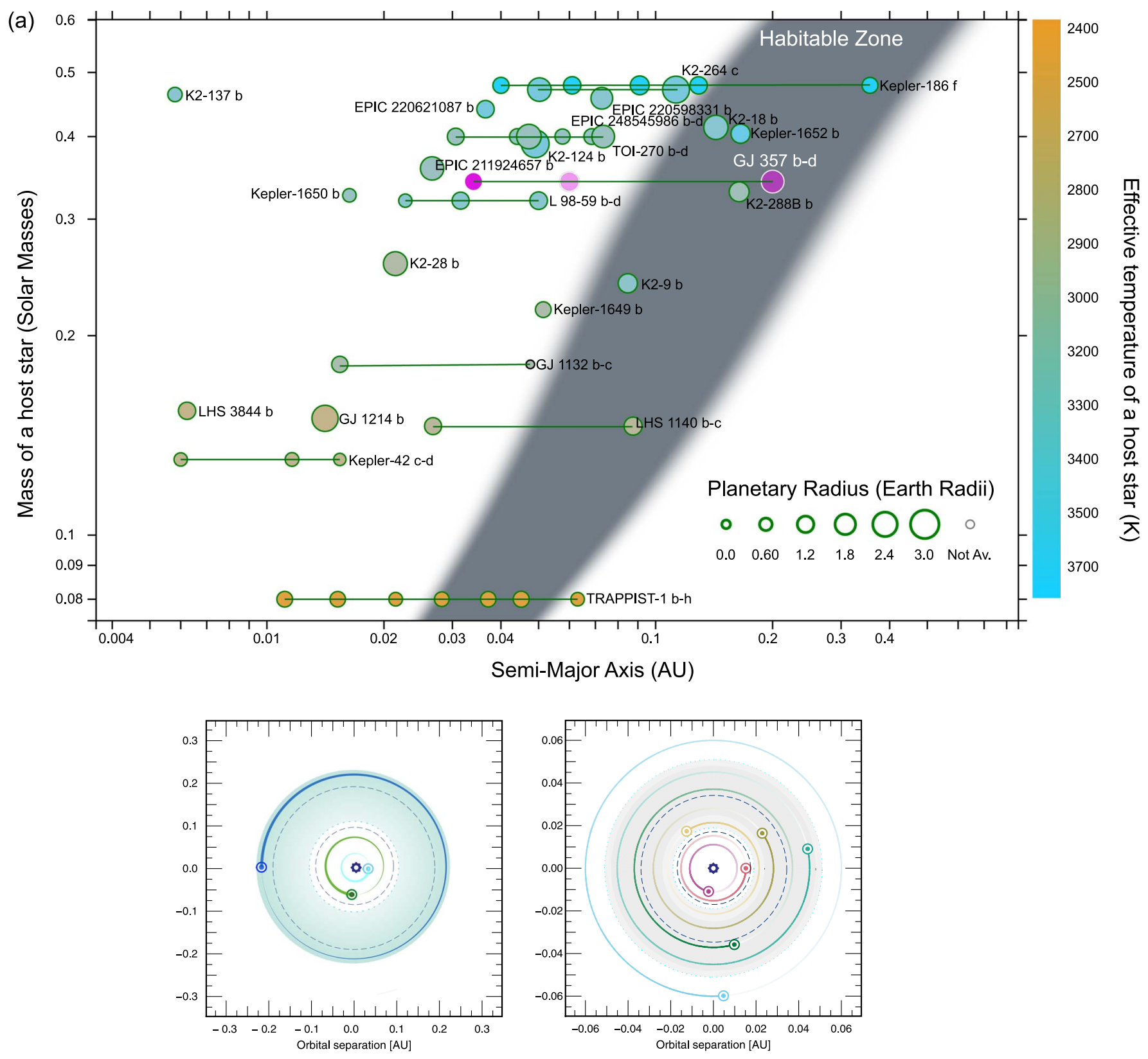

(b)

Figure 2. Top panel: detected M-star planet systems with transiting planets (data exoplanet.eu, 2019 June 20, radii below $3 R_{\text {Earth }}$ ), compared to the GJ 357 systemnote that only GJ 357b has been detected in transit so far. Bottom panels: comparison of the GJ 357 (left panel, three planets) and the Trappist-1 (right panel, seven planets) M-star planetary system. The gray shaded region shows the HZ. The dashed line in both figures in the bottom row shows the equivalent orbit of present-day Venus and Mars (Trappist-1 figure adapted from Gillon et al. 2017).

ocean, however it does not stipulate a liquid ocean surface. At that time the Sun was $\sim 8 \%$ less bright than today, yielding a solar flux equivalent equal to 1.76 present-day Solar irradiance at Earth's orbit $\left(S_{\text {Earth }}\right)$. The empirical outer edge for the HZ, the so-called Early Mars limit, is based on observations suggesting that Mars did not have liquid water on its surface at or before 3.8 billion years ago. At that time the solar flux was about $25 \%$ lower or equal to about $0.32 S_{0}$. The corresponding orbital distances in our solar system are 0.75 au (Recent Venus limit) and 1.77 au (Early Mars limit), respectively, for present solar luminosity, excludes present-day Venus and includes present-day Mars. Note that being in the HZ does not necessarily mean that a planet is habitable, and in-depth follow-up spectral observations of their atmospheres are needed in order to characterize planets and search for signs of life (see the review in Kaltenegger 2017).

Figure 2 shows the known transiting $M$ star planetary systems with planets with less than $3 R_{\text {Earth }}$ in terms of orbital semimajor axis of their planets as well as the contours of the HZ. It compares the GJ 357 planetary system with another well-known planet system with seven Earth-size planets, Trappist-1, which is at a similar distance from the Sun as GJ 357. It is another interesting target for observations because it harbors three planets in the HZ (Gillon et al. 2017) and an additional fourth planet in the Volcanic Habitable Zone (Ramirez \& Kaltenegger 2017). GJ 357 d $(0.38 \pm 0.01$ 
Table 1

Stellar and Planetary Parameters for GJ 357 System (Luque et al. 2019)

\begin{tabular}{|c|c|c|c|}
\hline \multirow{2}{*}{$\begin{array}{l}\text { Star } \\
\text { Name }\end{array}$} & \multicolumn{3}{|c|}{ GJ 357} \\
\hline & L $678-39$ & GJ 357 & Luyten (1942), Gliese (1957) \\
\hline & TOI-562 TESS Alerts & TIC 413248763 & Stassun et al. (2018) \\
\hline Spectral Type & \multicolumn{2}{|c|}{$\mathrm{M} 2.5 \mathrm{~V}$} & Hawley et al. (1996) \\
\hline \multirow[t]{3}{*}{ Brightness } & $B[\mathrm{mag}] 12.52 \pm 0.02$ & $V[\mathrm{mag}] 10.92 \pm 0.03$ & Fourth U.S. Naval Observatory CCD Astrograph Catalog (UCAC4) \\
\hline & $J[\mathrm{mag}] 7.337 \pm 0.034$ & $H[\mathrm{mag}] 6.740 \pm 0.0332$ & Two Micron All-Sky Survey (2MASS) \\
\hline & \multicolumn{2}{|c|}{$G[\mathrm{mag}] 9.8804 \pm 0.0014$} & Gaia second data release (DR2) \\
\hline Distance [mas] & \multicolumn{2}{|c|}{$105.88 \pm 0.06$} & Gaia DR2 \\
\hline Distance $[\mathrm{pc}]$ & \multicolumn{2}{|c|}{$9.444 \pm 0.005$} & Gaia DR2 \\
\hline Mass $\left[M_{\text {Sun }}\right]$ & \multicolumn{2}{|c|}{$0.342 \pm 0.011$} & Schweitzer et al. (2019) \\
\hline Radius $\left[R_{\text {Sun }}\right]$ & \multicolumn{2}{|c|}{$0.337 \pm 0.015$} & Schweitzer et al. (2019) \\
\hline$L\left[10^{-4} L_{\text {Sun }}\right]$ & \multicolumn{2}{|c|}{$159.1 \pm 3.6$} & Schweitzer et al. (2019) \\
\hline$T_{\text {eff }}[\mathrm{K}]$ & \multicolumn{2}{|c|}{$3505 \pm 51$} & Schweitzer et al. (2019) \\
\hline $\log g$ & \multicolumn{2}{|c|}{$4.94 \pm 0.07$} & Schweitzer et al. (2019) \\
\hline$[\mathrm{Fe} / \mathrm{H}]$ & \multicolumn{2}{|c|}{$0.12 \pm 0.16$} & Schweitzer et al. (2019) \\
\hline Planets & GJ 357 b & GJ 357 c & GJ 357 d \\
\hline$M_{\text {planet }}\left(M_{\text {Earth }}\right)$ & $1.84 \pm 0.31$ & $>3.40 \pm 0.46$ & $>6.1 \pm 1.0$ \\
\hline$R_{\text {planet }}\left(R_{\text {Earth }}\right)$ & $1.217 \pm 0.084$ & $\ldots$ & $\ldots$ \\
\hline$\left(\mathrm{g} / \mathrm{cm}^{-3}\right)$ & $5.6 \pm 1.7$ & $\cdots$ & $\cdots$ \\
\hline Inclination (deg) & $89.12 \pm 0.3$ & $\cdots$ & $\cdots$ \\
\hline$t_{\text {Transit }}(\mathrm{hr})$ & $1.53 \pm 0.1$ & $\cdots$ & $\cdots$ \\
\hline$a_{\text {planet }}(\mathrm{au})$ & $0.035 \pm 0.002$ & $0.061 \pm 0.004$ & $0.204 \pm 0.015$ \\
\hline$S_{\text {Earth }}$ & $12.6 \pm 1$ & $4.45 \pm 0.14$ & $0.38 \pm 0.01$ \\
\hline
\end{tabular}

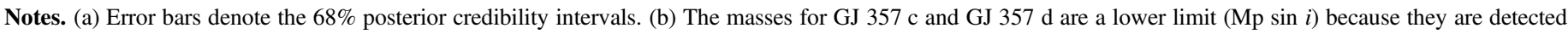
from radial velocities only. (c) References. Gaia DR2: Gaia Collaboration et al. (2018); UCAC4: Zacharias et al. (2013); 2MASS: Skrutskie et al. (2006).

$S_{\text {Earth }}$ ) receives comparable stellar irradiation to Trappist-1 $\mathrm{f}$ $\left(0.35 \pm 0.02 S_{\text {Earth }}\right)$ and also orbits in the outer part of the HZ.

Most exoplanets with small minimum masses orbit in the HZ of dim M dwarfs (e.g., Udry et al. 2007; Anglada-Escudé et al. 2013, 2016; Tuomi \& Anglada-Escudé 2013; Dittmann et al. 2017; Gillon et al. 2017). Thus the brightness of GJ 357 makes this system a very interesting target for observations and atmospheric characterization of the planets' atmospheres. If future observations with, for example, the CHaracterising ExOPlanet Satellite (CHEOPs; Broeg et al. 2013) can detect a transit of GJ $357 \mathrm{~d}$, it would become the closest transiting planet in the HZ, allowing for in-depth studies of its atmosphere. However, even if GJ 357 d does not transit, the brightness of its star makes this planet in the HZ of a close-by $\mathrm{M}$ star a prime target for ground- and space-based observations. For transiting terrestrial planets around the closest stars, the James Webb Space Telescope (JWST), scheduled for launch in 2021 (e.g., Gardner et al. 2006; Clampin et al. 2009; Deming et al. 2009; Kaltenegger \& Traub 2009; Barstow \& Irwin 2016), as well as upcoming ground-based telescopes (e.g., Snellen et al. 2013; Rodler \& Lopez-Morales 2014), might be able to detect biosignatures in a rocky planet's atmosphere for planets around the closest stars. The Extremely Large Telescopes (ELTs) will focus on observations in the visible but also have capabilities in the NIR to infrared (IR), such as the Midinfrared ELT Imager and Spectrograph (METIS) instrument at the ELT. Observations can also characterize planetary atmospheres if the planet does not transit their host star due to the known orbital movement and resulting radial velocity shift (see, e.g., Brogi et al. 2014).

Several space mission concepts to characterize Earth-like planets are currently being designed by, for example, NASA's science and technology definition teams, but no concept has been selected yet. Different concepts such as stars-shades and coronagraphs are designed to take spectra of extrasolar planets with the ultimate goal of remotely detecting atmospheric signatures to characterize nearby Super-Earths and Earth-like planets, enable comparative planetology beyond our solar system, and search for signs of life on other worlds.

Signs of life that modify the atmosphere or the surface of a planet, and thus can be remotely detectable, are key to finding life on exoplanets or exomoons (see, e.g., the review in Kaltenegger 2017). Observations of our Earth with its diverse biota function as a Rosetta Stone to identify habitats. Some atmospheric species exhibit noticeable spectral features in our planet's spectrum as a result, directly or indirectly, of biological activity: the main ones are $\mathrm{O}_{2}, \mathrm{O}_{3}, \mathrm{CH}_{4}, \mathrm{~N}_{2} \mathrm{O}$, and $\mathrm{CH}_{3} \mathrm{Cl}$. Any biosignature needs to be analyzed critically for potential geological sources under conditions that are different from those on Earth (see, e.g., Kasting et al. 2014; Kaltenegger 2017; Schwieterman et al. 2018; Walker et al. 2018). For prebiotic chemistry additional chemicals have been proposed as atmospheric signatures to look for (see, e.g., Ranjan et al. 2017; Rimmer et al. 2018). Spectroscopy can reveal the presence of specific molecules and atoms by identifying their characteristic energy transitions.

Section 2 discusses models, Section 3 shows results, and Section 4 discusses and concludes this Letter.

\section{Methods}

In this Letter we focus on GJ $357 \mathrm{~d}$, a planet with a minimum mass of $6.1 \pm 1$ Earth masses, $M_{\text {Earth }}$ (see Luque et al. 2019 and Table 1). This translates into a rocky planetary radius of $1.75 R_{\text {Earth }}$ assuming rocky composition and $2.4 R_{\text {Earth }}$ assuming pure ice composition, which is the limiting case for the largest core radius for a rocky planet (see Zeng \& Sasselov 2013 for details). This translates into a surface gravity of about twice Earth's surface gravity for a 1.75 Earth radius model, and 1.16 Earth's gravity for a 2.4 Earth radius model. 
We use the spectra model for the M2V active stars model (described in Rugheimer et al. 2015) as the host star input spectra, which has a similar effective surface temperature to GJ 357. The ultraviolet (UV) stellar spectrum is based on International Ultraviolet Explorer (IUE) data (see Rugheimer et al. 2015). We model four different types of atmospheres here for GJ $357 \mathrm{~d}$ for rocky composition, a radius of $1.75 R_{\text {Earth }}$ and a gravity of 2 times Earth's gravity as well as for a water-world composition, a radius of $2.4 R_{\text {Earth }}$, and a gravity of 1.16 times Earth's gravity.

Two atmospheric models assume Earth-analog outgassing rates for surface pressures of 1 and 2 bars. Two more models, one anoxic atmosphere and one oxic atmosphere, assume increased greenhouse effect from $\mathrm{CO}_{2} \mathrm{CH}_{4}$, and $\mathrm{H}_{2}$ concentrations added until the planet's average surface temperature is at least 273 for a 2 bar and 5 bar surface pressure.

For the two scenarios with Earth-analog outgassing ratios (see Rugheimer et al. 2015) but different surface pressures of 1 and 2 bars, we keep the planetary outgassing rates for $\mathrm{H}_{2}, \mathrm{CH}_{4}$, $\mathrm{CO}, \mathrm{N}_{2} \mathrm{O}$, and $\mathrm{CH}_{3} \mathrm{Cl}$ constant, and mixing ratios of $\mathrm{O}_{2}$ at 0.21 and $\mathrm{CO}_{2}$ at $3.55 \times 10^{-6}$, with a varying $\mathrm{N}_{2}$ concentration that is used as a fill gas to reach the set surface pressure of the model following Segura et al. (2005). Note that by keeping the outgassing rates constant, higher surface pressure atmosphere models initially have slightly lower mixing ratios of chemicals with constant outgassing ratios than lower surface pressure models.

We model an additional anoxic case for each planet core model, where we increase $\mathrm{CO}_{2}$ concentration to maintain an average surface pressure above freezing for the planetsassuming the planet also has a similar geological cycle to Earth's carbonate-silicate cycle, which stabilizes the surface temperature and regulates $\mathrm{CO}_{2}$ concentration in the atmosphere over geological timescales (see, e.g., Walker et al. 1981). This could provide liquid water and habitable surface conditions on the planet. For the anoxic atmospheres we assume a mixing ratio of $\mathrm{CO}_{2}$ at $0.1, \mathrm{CH}_{4}$ at 0.048 , and $\mathrm{H}_{2}$ at 0.16 . For the 1.75 Earth radii case this yields a surface temperature of $273 \mathrm{~K}$, and for the 2.4 Earth radii case this yields a surface temperature of $288 \mathrm{~K}$. We include $\mathrm{H}_{2}$ as an additional greenhouse gas due to the larger masses of these planets and its possible role in the heating of early Mars at a similar effective insolation (see e.g., Ramirez et al. 2014; Wordsworth et al. 2017; Ramirez \& Kaltenegger 2017; Wordsworth \& Pierrehumbert 2013; Wordsworth 2012).

The model for a warm oxic atmosphere for GJ $357 \mathrm{~d}$ employs an atmospheric mixing ratio of $0.1 \mathrm{O}_{2}$ and a biotic methane flux of $8.57 \times 10^{10}$ molecules $\mathrm{cm}^{-2} \mathrm{~s}^{-1}$, which is consistent with a $0.1 \mathrm{O}_{2}$ atmosphere during the Proterozoic period (Olson et al. 2016). A mixing ratio of $0.8 \mathrm{CO}_{2}$ and a surface pressure of 5 bars results in a surface temperature of $288 \mathrm{~K}$. Note that different atmospheric compositions (e.g., different mixing ratios of $\mathrm{CO}_{2}, \mathrm{CH}_{4}$, and $\mathrm{H}_{2}$ ) can maintain temperatures for liquid water on the surface of GJ $357 \mathrm{~d}$ and our model only shows one possibility for reference.

For this study, we use a 1D climate and photochemistry code, EXO-Prime, a coupled 1D radiative-convective atmosphere code developed for rocky exoplanets coupled to a lineby-line radiative transfer code, which generates the spectra in different viewing geometries (see, e.g., Kaltenegger \& Traub 2009; Kaltenegger \& Sasselov 2010; Rugheimer et al. 2013, 2015; Rugheimer \& Kaltenegger 2018) to model the
Earth-like atmospheres and a 1D atmospheric model developed for the study of the habitability of GJ581 d (see, e.g., Kaltenegger et al. 2011).

EXO-Prime simulates both the effects of stellar radiation on a planetary environment and the planet's outgoing spectrum. We model an altitude range in the atmosphere that extends upward to a minimum of $70 \mathrm{~km}$ with 50 height layers. We use a geometrical model in which the average 1D global atmospheric model profile is generated using a plane-parallel atmosphere, treating the planet as a Lambertian sphere, and setting the stellar zenith angle to $60^{\circ}$ to represent the average incoming stellar flux on the dayside of the planet (see also Schindler \& Kasting 2000). The temperature in each layer is calculated from the difference between the incoming and outgoing flux and the heat capacity of the atmosphere in each layer. If the lapse rate of a given layer is larger than the adiabatic lapse rate, it is adjusted to the adiabatic rate until the atmosphere reaches equilibrium.

We use a two-stream approximation (see Toon et al. 1989), which includes multiple scattering by atmospheric gases, in the visible/NIR to calculate the shortwave fluxes. Four-term, correlated-k coefficients parameterize the absorption by $\mathrm{O}_{3}$, $\mathrm{H}_{2} \mathrm{O}, \mathrm{O}_{2}$, and $\mathrm{CH}_{4}$ (Kasting \& Ackerman 1986). A fixed relative humidity is assumed following Manabe \& Wetherald (1967). The tropospheric lapse rate follows a moist adiabat (Kasting \& Ackerman 1986) that takes into account $\mathrm{CO}_{2}$ and $\mathrm{H}_{2} \mathrm{O}$ condensation. For all the models $\mathrm{N}_{2}$ concentration is calculated to fill out the atmosphere after the concentrations of the other chemical species have been set up. In the thermal IR region, a rapid radiative transfer model calculates the longwave fluxes. Clouds are not explicitly calculated. The photochemistry code, originally developed by Kasting et al. (1984), solves for 55 chemical species linked by 220 reactions using a reverseEuler method (see Segura et al. 2010, and references therein). The anoxic atmosphere model is explained in Segura et al. (2007), Haqq-Misra et al. (2008), and Kaltenegger et al. (2011).

The photochemical model is stationary, and convergence is achieved when the following criteria are fulfilled: the production and loss rates of chemical species are balanced, which results in a steady state for the chemical concentrations, and the initial boundary conditions, such a surface mixing ratios or surface fluxes, are met. Photolysis rates for various gas-phase species are calculated using a $\delta$ two-stream routine (Toon et al. 1989) that accounts for multiple scattering by atmospheric gases and by sulfate and hydrocarbon aerosols. One important feature of the high $\mathrm{CO}_{2}$ model is its ability to keep track of the atmospheric hydrogen budget, or redox budget. $\mathrm{H}$ and $\mathrm{H}_{2}$ escape was simulated by assuming an upward flux at the diffusion limited rate (Walker 1977).

The radiative transfer model used to compute planetary spectra is based on a model that was originally developed for trace gas retrieval in Earth's atmospheric spectra (Traub \& Stier 1976) and further developed for exoplanet transmission and emergent spectra (Kaltenegger et al. 2007; Kaltenegger \& Traub 2009; Kaltenegger 2010; Kaltenegger \& Sasselov 2010; Kaltenegger et al. 2013). In this Letter, we model Earth's transmission, and reflected and thermal emission spectra using 21 of the most spectroscopically significant molecules $\left(\mathrm{H}_{2} \mathrm{O}\right.$, $\mathrm{O}_{3}, \mathrm{O}_{2}, \mathrm{CH}_{4}, \mathrm{CO}_{2}, \mathrm{OH}, \mathrm{CH}_{3} \mathrm{Cl}, \mathrm{NO}_{2}, \mathrm{~N}_{2} \mathrm{O}, \mathrm{HNO}_{3}, \mathrm{CO}, \mathrm{H}_{2} \mathrm{~S}$, $\mathrm{SO}_{2}, \mathrm{H}_{2} \mathrm{O}_{2}, \mathrm{NO}, \mathrm{ClO}, \mathrm{HOCl}, \mathrm{HO}_{2}, \mathrm{H}_{2} \mathrm{CO}, \mathrm{N}_{2} \mathrm{O}_{5}$, and $\mathrm{HCl}$ ).

For the reflected and emitted spectra, we use a Lambert sphere as an approximation for the disk-integrated planet in our 
model. The surface of our model planet corresponds to Earth's current surface of $70 \%$ ocean, $2 \%$ coast, and $28 \%$ land. The land surface consists of $30 \%$ grass, $30 \%$ trees, $9 \%$ granite, $9 \%$ basalt, $15 \%$ snow, and $7 \%$ sand. Surface reflectivities are taken from the USGS Digital Spectral Library and the ASTER Spectral Library (following Kaltenegger et al. 2007). For our larger water worlds we assume a similar overall surface albedo for ease of comparison between our models. Note that the change in surface albedo between a liquid water surface (low albedo and stronger absorption of incoming irradiation) or a pristine frozen world (high reflectivity), would in addition influence the planet's climate. However, it is an ongoing scientific discussion on how pristine an icy surface would remain on such a world. Without further input, we chose to maintain a similar overall surface albedo of about 0.16 for all our models.

For the transmission spectrum, we trace the light from the star through individual layers in the atmosphere and then combined the spectra to the overall transmission spectra of the planet as discussed in detail in Kaltenegger \& Traub (2009) and Bétrémieux \& Kaltenegger (2014).

We calculate the spectrum at high spectral resolution with several points per line width. The line shapes and widths are computed using Doppler and pressure broadening on a line-byline basis for each layer in the model atmosphere. The overall high-resolution spectrum is calculated with $0.1 \mathrm{~cm}^{-1}$ wavenumber steps. The figures are shown smoothed to a resolving power of 700 using a triangular smoothing kernel. The spectra may be binned further for comparison with proposed future spectroscopy missions designs to characterize Earth-like planets. We previously validated EXO-Prime from the visible to the IR using data from ground and space (see e.g., Kaltenegger et al. 2007; Kaltenegger \& Traub 2009; Rugheimer et al. 2013).

All of oxygenic simulations used a fixed mixing ratio of $355 \mathrm{ppm}$ for $\mathrm{CO}_{2}$ and $21 \% \mathrm{O}_{2}$. For the sample anoxic model we set $\mathrm{CO}_{2}=1.0 \times 10^{-1}$ and $\mathrm{H}_{2}$ to $1.9 \times 10^{-4}$. The $\mathrm{N}_{2}$ mixing ratio is set to be a fill gas such that the total surface pressure is 1 or 2 bars with a fixed upper boundary of $10^{-7}$ bar (a minimum of $70 \mathrm{~km}$ ).

\section{Results}

GJ $357 \mathrm{~d}$ has a minimum mass of $6.1 \pm 1 M_{\text {Earth }}$ (see Luque et al. 2019). Assuming the minimum mass is the real planet's mass, this translates into a rocky planetary radius of $1.75 R_{\text {Earth }}$, assuming rocky composition and $2.4 R_{\text {Earth }}$ assuming pure ice composition, which is the limiting case for the largest core radius for a rocky planet (see Zeng \& Sasselov 2013 for details). This translates into a surface gravity of about twice Earth's surface gravity for a 1.75 Earth radius model and 1.16 Earth's gravity for a $2.4 R_{\text {Earth }}$ model. GJ 357 d receives 0.38 times Earth's irradiation.

We model three different types of atmospheres here for GJ $357 \mathrm{~d}$ for (i) a rocky composition, with a radius of $1.75 R_{\text {Earth }}$ and a gravity of 2 times Earth's gravity, as well as (ii) a waterworld composition, with a radius of $2.4 R_{\text {Earth }}$ and a gravity of 1.16 times Earth's gravity. These two atmospheric models assume Earth-analog outgassing rates for surface pressure of 1 bar and 2 bars. We also model an anoxic atmosphere as a third example, where we increase $\mathrm{CO}_{2}$ concentration to a mixing ratio of $10^{-1}$, under which the planet's average surface temperature is above freezing.
GJ 357 d received similar flux to Mars in our own solar system. However, it is more massive than Mars, and if we assume geological activity similar to Earth, an increase in atmospheric $\mathrm{CO}_{2}$ at lower stellar irradiation is expected. On a geologically active planet like Earth the geochemical carbonate-silicate cycle stabilizes the long-term climate and atmospheric $\mathrm{CO}_{2}$ content, depending on the surface temperature: $\mathrm{CO}_{2}$ is continuously outgassed and forms carbonates in the presence of surface water, which then get subducted and $\mathrm{CO}_{2}$ gets outgassed again. Farther from the star, the lower stellar irradiance would create a cooler surface temperature on a planet, thus linking the orbital distance to atmospheric $\mathrm{CO}_{2}$ concentrations levels: $\mathrm{CO}_{2}$ should be a trace gas close to the inner edge of the $\mathrm{HZ}$ but a major compound in the outer part of the $\mathrm{HZ}$ with several bars of $\mathrm{CO}_{2}$ (e.g., Walker 1981). Because the outer limit of the $\mathrm{HZ}$ is based on the assumption that atmospheric $\mathrm{CO}_{2}$ will build up and warm the planet, an adequate $\mathrm{CO}_{2}$ mixing ratio in the atmosphere is needed to maintain surface temperatures above freezing for planets on the outer part of the $\mathrm{HZ}$. We increase $\mathrm{CO}_{2}$ levels in our anoxic model runs, to a mixing ratio to $10^{-1}$, which provides surface conditions above freezing for both planet models, a rocky core composition, as well as an icy core composition.

The average surface temperature for the sample models are $211 \mathrm{~K}$ ( 1 bar, $\left.1.75 R_{\text {Earth }}\right), 215 \mathrm{~K}$ ( 2 bars, $\left.1.75 R_{\text {Earth }}\right), 215 \mathrm{~K}$ (1 bar, $2.4 R_{\text {Earth }}$ ), $221 \mathrm{~K}$ (2 bars, $\left.2.4 R_{\text {Earth }}\right)$ for the models assuming Earth-like outgassing rates without an increase in $\mathrm{CO}_{2}$. For our anoxic atmosphere model with an increase in $\mathrm{CO}_{2}$ and $\mathrm{H}_{2}$ levels the average surface temperature is $273 \mathrm{~K}$ ( 2 bars,

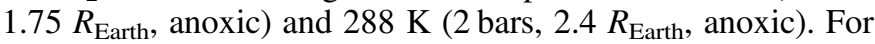
our oxic high $\mathrm{CO}_{2}$ atmosphere the surface temperature is $278 \mathrm{~K}$ (5 bars, $1.75 R_{\text {Earth }}$, oxic).

Figure 3 shows the temperature as well as water, ozone, methane, and $\mathrm{H}_{2}$ mixing ratios versus height in the model atmospheres for GJ $357 \mathrm{~d}$. Note that some oxygen and ozone also builds up in the atmosphere of terrestrial planets around $\mathrm{M}$ stars without a biological source, as expected (see e.g., Hu et al.

2012; Domagal-Goldman et al. 2014; Wordsworth \& Pierrehumbert 2014).

\section{Model Spectra for GJ 357 d}

Encoded in the planet's transmission, reflection, and emission spectra is the information of the chemical makeup of a planet's atmosphere and the atmosphere's transparency; the emergent spectrum also carries some information about surface properties for emergent flux. This makes light from a planet a crucial tool with which to characterize it. The presence or absence of spectral features will indicate similarities or differences of the atmospheres of terrestrial exoplanets from Earth, and their astrobiological potential.

We show synthetic spectra for upcoming space and ground telescopes in Figures 4 and 5 with a spectral resolution $(\lambda / \Delta \lambda)$ of 700 as a sample resolution, like the high-resolution setting for the Near InfraRed Spectrograph (NIRSpec) instrument on JWST. While different spectral resolution is envisioned for different instruments, 700 gives a good sample overview of how the spectral features will appear. We keep the spectral resolution constant for the spectra shown in the figures to allow easy cross comparison and indicate some commonly used filters in Astronomy. Note that all spectra are available online (carlsaganinstitute.org/data) in high resolution (run at $0.1 \mathrm{~cm}^{-1}$ wavenumber, which corresponds to a spectral resolution $(\lambda /$ 

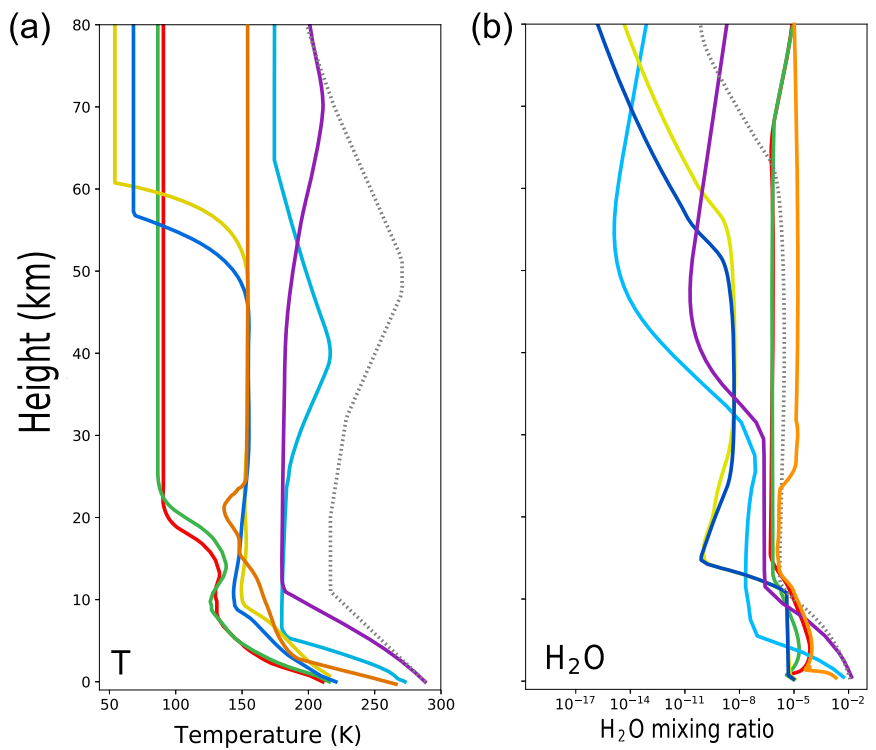

(c)

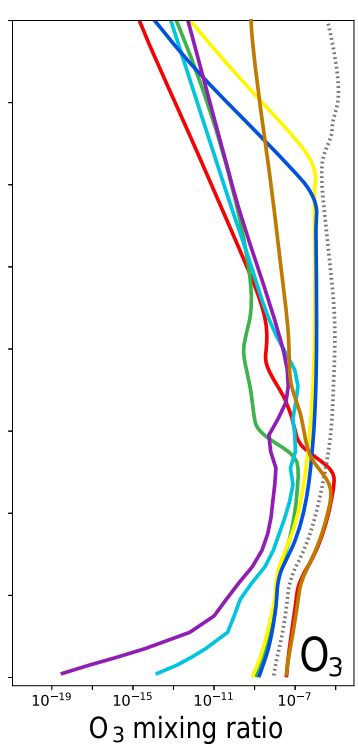

(d)

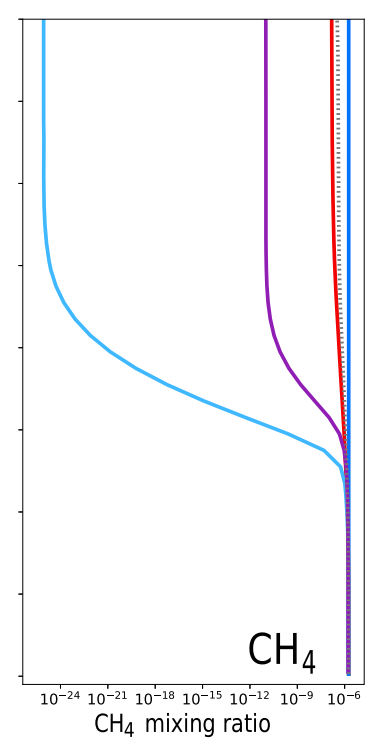

(e)

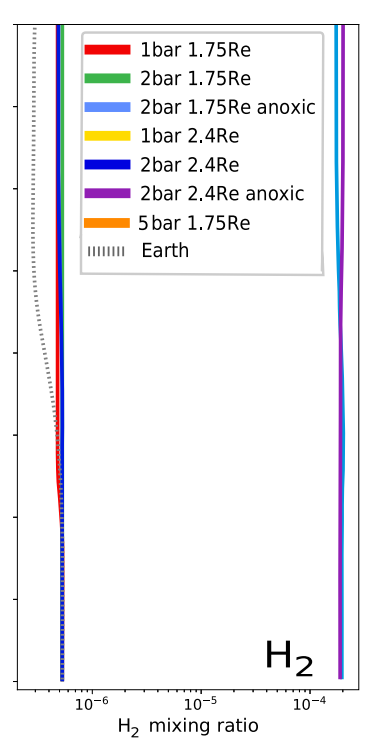

Figure 3. Temperature and chemical mixing ratios of water, ozone, methane, and hydrogen in the GJ $357 \mathrm{~d}$ sample atmosphere models vs. height.
$\Delta \lambda)$ of 100,000 at $1 \mu \mathrm{m})$. The high-resolution spectra can then be smeared to any required resolution.

Several teams have discussed how to observe spectral features of temperate rocky planets (e.g., Kaltenegger \& Traub 2009; Pallé et al. 2009; Snellen et al. 2013; Morley et al. 2017); a full model for the different instruments available on JWST as well as the ELTs should be run once it is determined whether the planet transits, which would constrain the radius and thus the gravity and models for the planet.

\section{Reflection and Emission Spectra for GJ 357 d}

Figure 4 shows the reflected flux (top panel) and (bottom panel) emission spectra for our models for GJ $357 \mathrm{~d}$ as sample spectra for JWST as well as ELTs for direct imaging or secondary eclipse measurements. We include the relative reflection spectra (Figure 4, middle panel) here to show the effect of the incident starlight on the detectable chemical species. As for any spectral features, the amount of chemicals needed to show a feature varies depending on the geometry of observations as well as atmospheric composition and temperature.

The depth of spectral features in reflected light is dependent on the abundance of a chemical as well as the incoming stellar radiation at that wavelength. The sample spectra for GJ $357 \mathrm{~d}$ shows strong atmospheric features in reflected light (Figure 4, top panel) from 0.7 to $4 \mu \mathrm{m}$, especially for the warmer anoxic sample atmospheres for $\mathrm{H}_{2} \mathrm{O}$ at e.g., 1.4 and $1.9 \mu \mathrm{m}$, and a smaller $\mathrm{CH}_{4}$ features at $1.7 \mu \mathrm{m}$, and $2.4 \mu \mathrm{m}$, Earth-like atmospheres show $\mathrm{O}_{2}$ at $0.76 \mu \mathrm{m}$. The region between 2 and 5 microns is not shown in Figure 4 because the flux from the planet in reflection and emission is extremely low.

In thermal emission, the depth of spectral features depends on the abundance of a chemical as well as the temperature difference between the emitting/absorbing layer and the continuum. In the IR, the strongest atmospheric features in our models for GJ $357 \mathrm{~d}$ from 4 to $20 \mu \mathrm{m}$ are $\mathrm{O}_{3}$ at $9.6 \mu \mathrm{m}$, $\mathrm{CO}_{2}$ at $15 \mu \mathrm{m}, \mathrm{H}_{2} \mathrm{O}$ at $6.4 \mu \mathrm{m}$ and $17 \mu \mathrm{m}$, and $\mathrm{CH}_{4}$ at $7.7 \mu \mathrm{m}$. The IR shows the difference in surface temperature of the planet models strongly with both anoxic models being run with $\mathrm{CO}_{2}$ concentrations that warm the planet above freezing compared to the other cooler surface, thus showing a larger emission than the planet models with colder surfaces. Therefore, in order to see the features of the cooler planets we did not include the hotter anoxic models in Figure 4 (they are instead available online). As shown in Figure 4, high amounts of $\mathrm{CO}_{2}$ dominate the IR emission spectrum, as previously shown in model spectra for a warm habitable Gl $581 \mathrm{~d}$ (Kaltenegger et al. 2011). The low overall flux also shows that the observable layer is not the surface, which would be a hotter blackbody temperature; consequently, the atmosphere cannot be probed to the ground for the high $\mathrm{CO}_{2}, 5$ bar atmospheric model. Thus, the ozone feature in the emission spectra is not detectable in this model, but the oxygen feature in the reflection spectra can still be observed (see also Kaltenegger et al. 2011).

Note that for both reflected light and emitted light we did not show the influence of planet size on the overall flux; this was so we could compare the spectra in one figure. However, the overall flux of the planet model assumes that ice composition for the core is about twice as large as the model for the rocky core, due to the difference in corresponding radius $\left(1.75 R_{\text {Earth }}\right.$ versus $\left.2.4 R_{\text {Earth }}\right)$. Planets with radius $2.4 R_{\text {Earth }}$ versus 1.75 $R_{\text {Earth }}$ have a larger area and thus will appear brighter. 

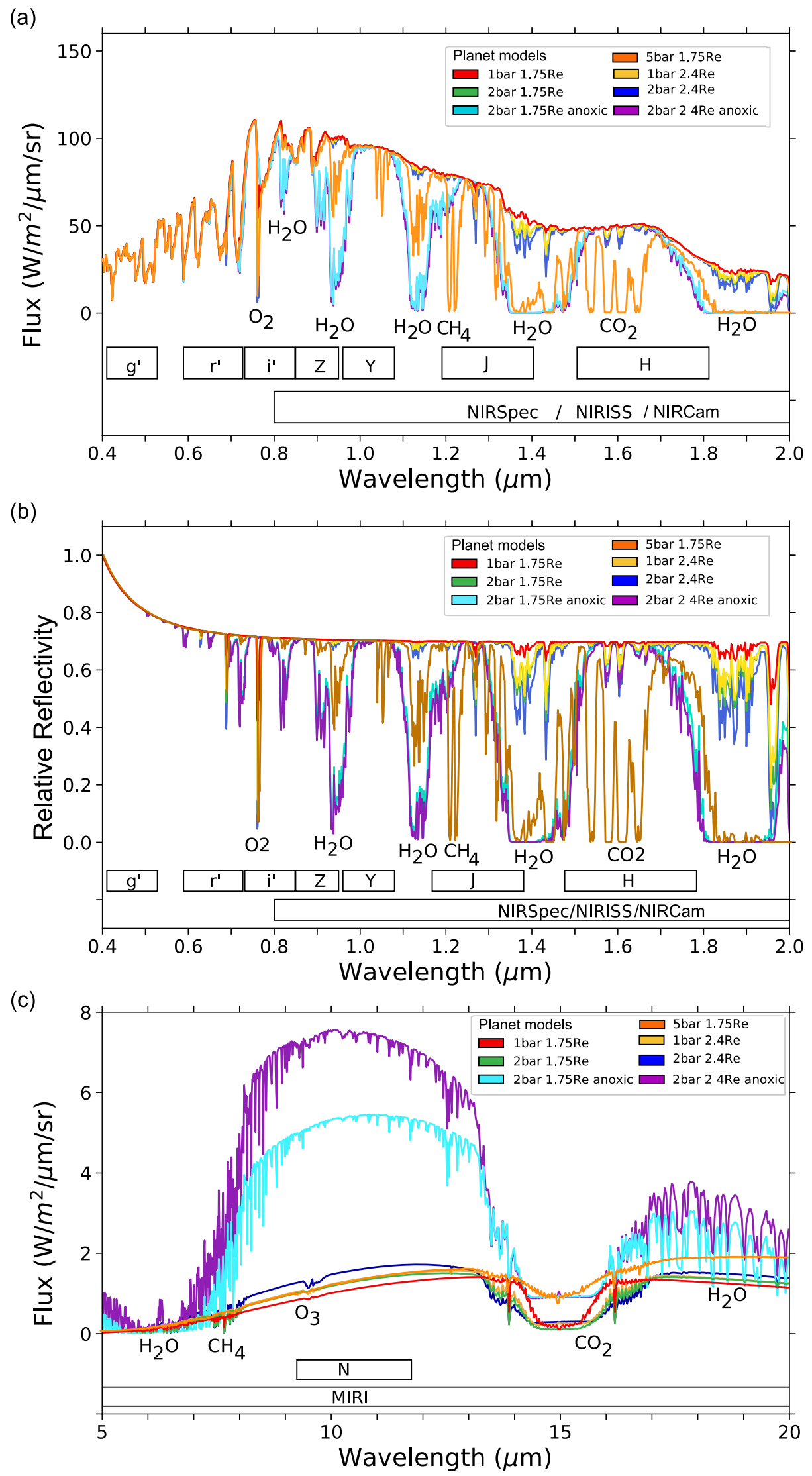

Figure 4. Flux of sample model spectra of reflected (top panel), relative reflection (middle panel) and emitted (bottom panel) of the planet models. Note that the size of the planet is not taken into account and thus the 2.4 and $1.75 R_{\text {Earth }}$ planets show similar flux levels. Spectra data for this figure is available online.

(The data used to create this figure are available.) 
(a)

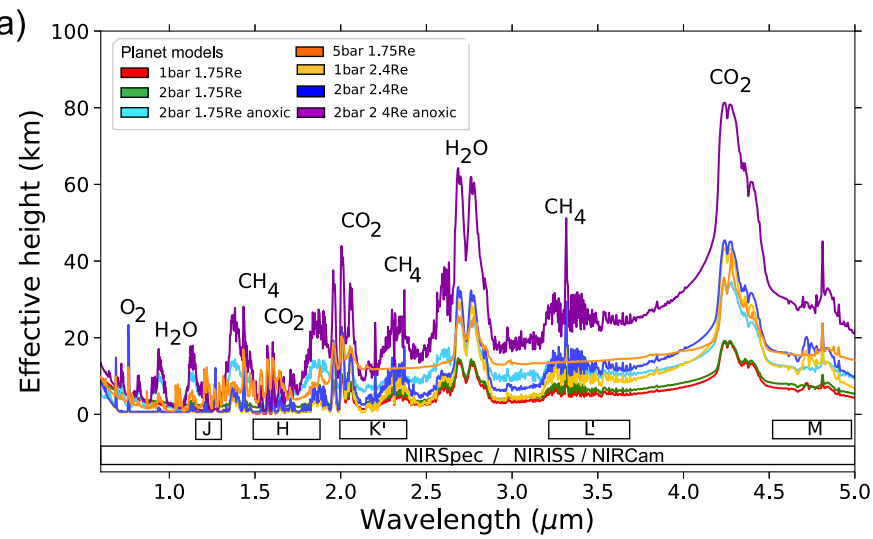

(c)
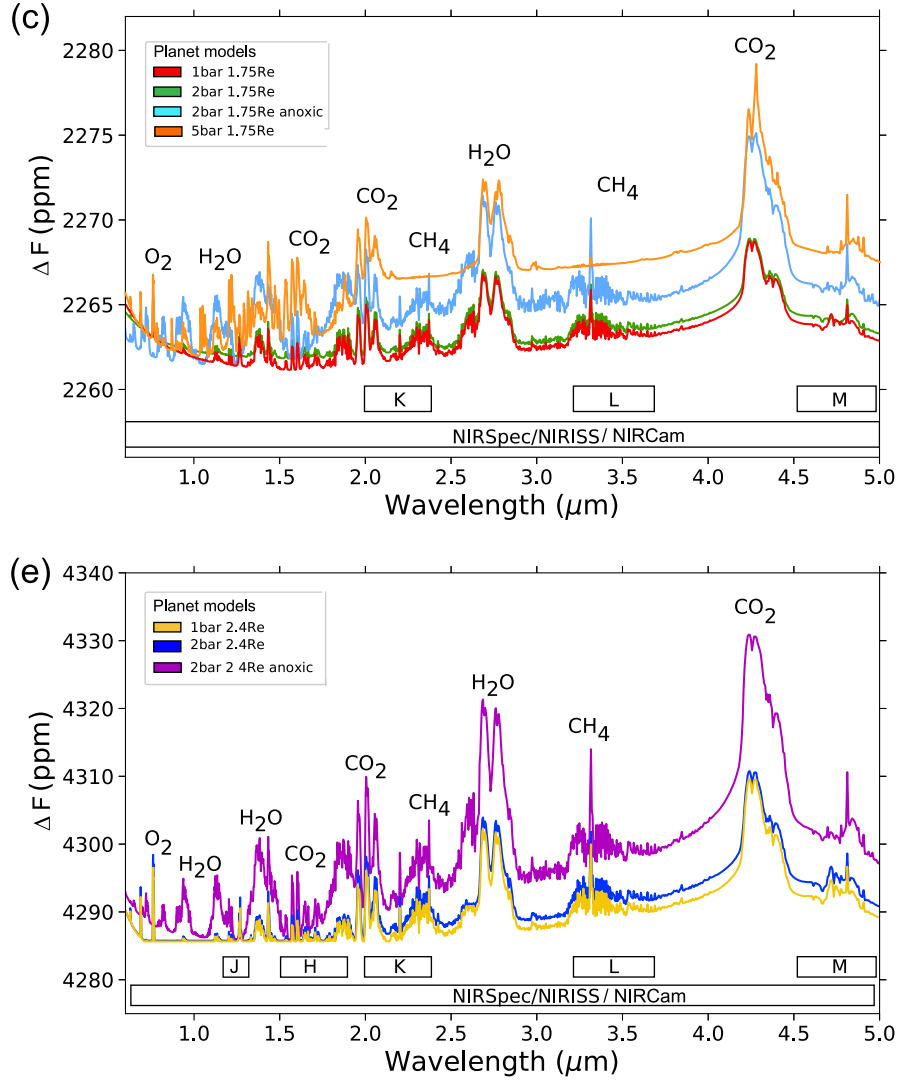

(b)

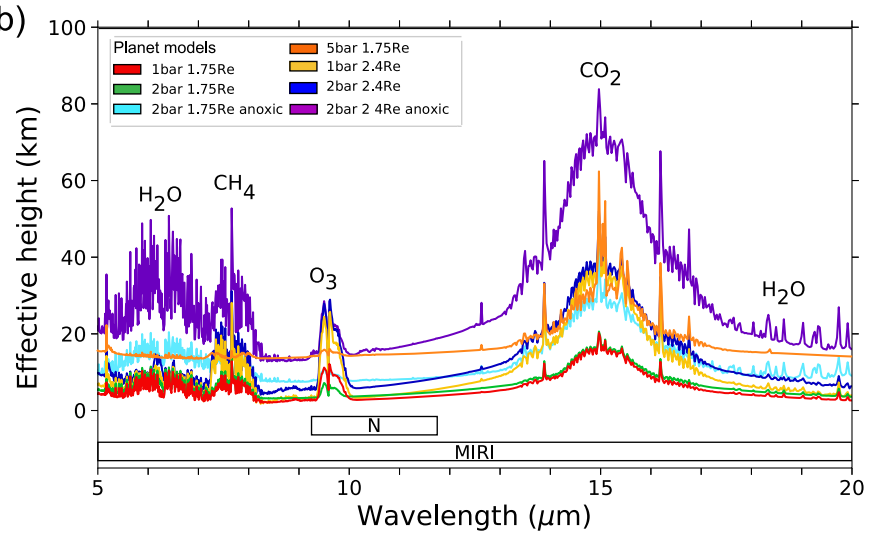

(d)

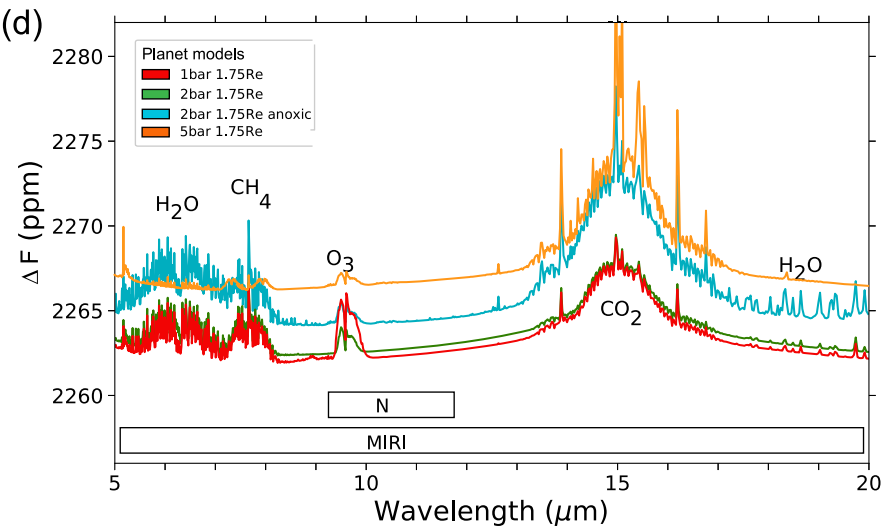

(f)

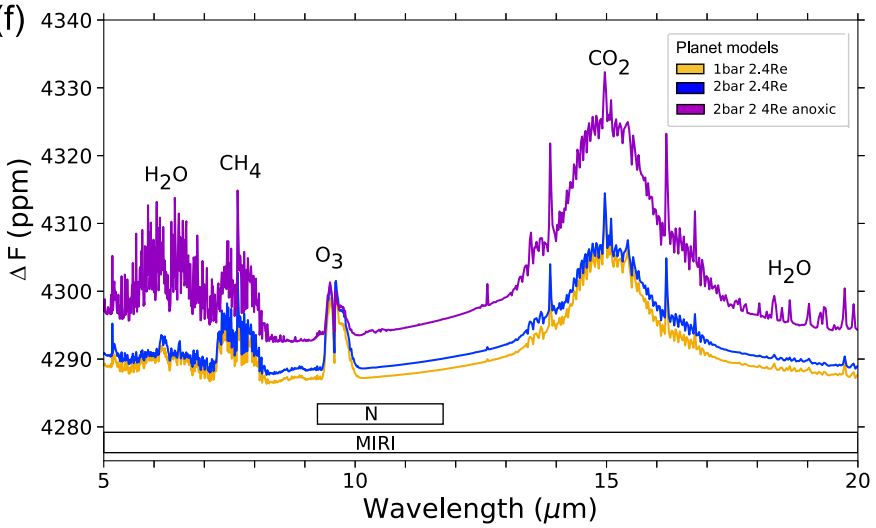

Figure 5. Flux difference (radius planet/radius star) ${ }^{2}$ in ppm transmission spectra of models of GJ $357 \mathrm{~d}$ in the $J W S T$ wavelength range for seven transmission spectra: this includes four atmosphere models and two interior models (top row) of effective atmospheric height, and the flux signal in ppm (the middle row for $1.75 R_{\text {Earth }}$ models, and the bottom row for $2.4 R_{\text {Earth }}$ models). Spectra data for this figure is available online.

(The data used to create this figure are available.)

For an Earth-like biosphere, the main detectable atmospheric chemical signatures that in combination could indicate habitability are $\mathrm{O}_{2} / \mathrm{O}_{3}$ in combination with $\mathrm{CH}_{4}$ or $\mathrm{N}_{2} \mathrm{O}$, and $\mathrm{CH}_{3} \mathrm{Cl}$. Reflection, as well as the emission spectra of GJ $357 \mathrm{~d}$, show both features for Earth-like atmospheres (see, e.g., reviews by Des Marais et al. 2002; Kaltenegger 2017; Schwieterman et al. 2018).

\section{Transmission Spectra for GJ 357 d}

Even though no transit for GJ $357 \mathrm{~d}$ has been detected yet, we also model transmission spectra for our model cases. The gravity, mean molecular weight, and temperature of a planet affect its transmission spectrum through the pressure profile of its atmosphere, and hence its atmospheric absorption profile. For an ideal gas atmosphere in hydrostatic equilibrium, the pressure, $p$, varies with the altitude, $z$, as $d \ln (p)=-1 / H d z$, where $H$ is the atmospheric scale height defined as $H=k T / \mu g$, $k$ is Boltzmann's constant, and $T, \mu$, and $g$ are the local (i.e., altitude dependent) temperature, mean molecular mass, and gravity, respectively (Figure 5, top row). The transit signal, the flux difference, $\Delta F$, depends in addition on the ratio of the planet's radius divided by the star's radius squared (Figure 5, middle and bottom rows).

Figure 5 (top row) shows the comparison of the effective height of the atmosphere in transit for our model planets in the top panels: increasing the gravity of the planet (twice Earth's 
gravity for a radius of $1.75 R_{\text {Earth }}$ versus 1.16 time Earth's gravity for a $2.4 R_{\text {Earth }}$ ) translates into a reduced transmission height. Increasing temperature increases the scale height and transmission signal; note that the anoxic cases for GJ $357 \mathrm{~d}$ were run to produce a surface temperature above freezing. This generates a warmer surface as well as atmospheric temperature due to increased greenhouse warming than in the Earth-like models, where we did not increase greenhouse gases above present-day Earth.

Figure 5 shows that the largest signal is generated by the lower gravity model of the $2.4 R_{\text {Earth }}$ planet, with the largest signal being generated by the warmest, denser 2 bar surface pressure atmosphere. All $2.4 R_{\text {Earth }}$ atmospheres at similar surface pressure produce larger signals than the $1.75 R_{\text {Earth }}$ models, because of the lower gravity assumed for the $2.4 R_{\text {Earth }}$ models.

The sample transit spectra for GJ 357 d shows strong atmospheric features in reflected light (Figure 5, top row) from 0.7 to $5 \mu \mathrm{m}$, especially for the warmer anoxic and oxic sample atmospheres for $\mathrm{H}_{2} \mathrm{O}$ at e.g., 1.4 and $1.9 \mu \mathrm{m}$, and a smaller $\mathrm{CH}_{4}$ features at $1.7 \mu \mathrm{m}$, and $2.4 \mu \mathrm{m}$. Oxic atmospheres show $\mathrm{O}_{2}$ at $0.76 \mu \mathrm{m}$. In the IR, the strongest atmospheric features in our models for GJ $357 \mathrm{~d}$ from 5 to $20 \mu \mathrm{m}$ are $\mathrm{CO}_{2}$ at $15 \mu \mathrm{m}, \mathrm{H}_{2} \mathrm{O}$ at $6.4 \mu \mathrm{m}$ and $17 \mu \mathrm{m}$ and $\mathrm{CH}_{4}$ at $7.7 \mu \mathrm{m}$. For the Earth-like atmospheres containing oxygen, the $\mathrm{O}_{3}$ at $9.6 \mu \mathrm{m}$ is visible. Note that due to the small size of its host star compared to the planet, assuming a rocky composition, the flux difference is favorable for future observations.

\section{Conclusions and Discussion}

We present planetary models as well as synthetic transmission, reflection, and emission spectra for a range of atmospheres for the newly discovered planet GJ $357 \mathrm{~d}$, which orbits in the HZ of its host star. The host star harbors two more known planets; the innermost is a confirmed transiting planet with a mean density that is similar to Earth. While it is still unknown if GJ $357 \mathrm{~d}$ transits, the brightness of its host star makes this planet in the $\mathrm{HZ}$ of a close-by $\mathrm{M}$ star a prime target for ground- and space-based atmosphere characterization. Assuming the minimum mass of GJ $357 \mathrm{~d}$ is the real planet's mass, this translates into a rocky planetary radius of $1.75 R_{\text {Earth }}$ assuming rocky composition, and $2.4 R_{\text {Earth }}$ assuming pure ice composition, which is the limiting case for the largest core radius for a rocky planet. We model under what conditions GJ $357 \mathrm{~d}$ could sustain liquid water and surface habitability for a range of different atmospheric conditions from Earth-like to anoxic atmospheres, for rocky to water worlds.

GJ $357 \mathrm{~d}$ receives 0.38 times Earth's irradiation, which is similar to Mars in our own solar system. We model three different types of atmospheres here for GJ $357 \mathrm{~d}$ for (i) a rocky composition and (ii) a water world composition. For Earthanalog outgassing rates for different surface pressures from 1 to 2 bars, the surface temperature remains below freezing. However, geological active worlds, like our Earth, are expected to build up $\mathrm{CO}_{2}$ concentrations due to the feedback of the carbonate-silicate cycle. We model oxic and anoxic atmospheres as three examples, where we increase $\mathrm{CO}_{2}$ concentration, so that the planet's average surface temperature is above freezing.

The sample reflection, emission, and transmission spectra show features of a wide range of chemicals, $\mathrm{H}_{2} \mathrm{O}, \mathrm{CO}_{2}, \mathrm{CH}_{4}$, and $\mathrm{O}_{3}$ and $\mathrm{O}_{2}$ for Earth-like atmospheres from the visible to IR wavelengths $(0.4-20 \mu \mathrm{m})$, which would indicate habitability for observations with upcoming telescopes.

\section{ORCID iDs}

J. Madden (i) https://orcid.org/0000-0002-4701-7833

N. Espinoza (1) https://orcid.org/0000-0001-9513-1449

\section{References}

Anglada-Escudé, G., Amado, P. J., Barnes, J., et al. 2016, Natur, 536, 437 Anglada-Escudé, G., Tuomi, M., Gerlach, E., et al. 2013, A\&A, 556, A126 Barstow, J. K., \& Irwin, P. G. J. 2016, MNRAS: Letters, 461, L92 Batalha, N. M. 2014, PNAS, 111, 12647

Bétrémieux, Y., \& Kaltenegger, L. 2014, ApJ, 791, 7

Broeg, C., Fortier, A., Ehrenreich, D., et al. 2013, EPJWC, 47, 03005

Brogi, M., de Kok, R. J., Birkby, J. L., Schwarz, H., \& Snellen, I. A. G. 2014, A\&A, 565, A124

Clampin, M., JWST Science Working GroupJWST Science Working GroupJWST Transits Working Group, Deming, D., \& Lindler, D. 2009, Comparative Planetology: Transiting Exoplanet Science with JWST, AST2010 Science Whitepaper JWST Transit Science, https://sci.esa.int/ documents/34594/36271/1567258178742-JWST_planetology.pdf

Deming, D., Seager, S., Winn, J., et al. 2009, PASP, 121, 952

Des Marais, D. J., Harwit, M. O., Jucks, K. W., et al. 2002, AsBio, 2, 153

Dittmann, J. A., Irwin, J. M., Charbonneau, D., et al. 2017, Natur, 544, 333

Domagal-Goldman, S. D., Segura, A., Claire, M. W., Robinson, T. D., \& Meadows, V. S. 2014, ApJ, 792, 90

Donahue, T. M., \& Pollack, J. B. 1983, in Venus, ed. D. M. Hunten (Tucson, AZ: Univ. Arizona Press), 1003

Dumusque, X., Pepe, F., Lovis, C., et al. 2012, Natur, 491, 207

Gaia Collaboration, Brown, A. G. A., Vallenari, A., et al. 2018, A\&A, 616, A1 Gardner, J. P., Mather, J. C., Clampin, M., et al. 2006, SSRv, 123, 485

Gillon, M., Triaud, A. H. M. J., Demory, B.-O., et al. 2017, Natur, 542, 456 Gliese, W. 1957, MiARI, 8, 1

Haqq-Misra, J., Domagal-Goldman, S. D., Kasting, P. J., \& Kasting, J. F. 2008, AsBio, 8, 1127

Hawley, S. L., Gizis, J. E., \& Reid, I. N. 1996, AJ, 112, 2799

Hu, R., Seager, S., \& Bains, W. 2012, ApJ, 761, 166

Kaltenegger, L. 2017, ARA\&A, 55, 433

Kaltenegger, L. 2010, ApJL, 712, L125

Kaltenegger, L., \& Haghighipour, N. 2013, ApJ, 777, 165

Kaltenegger, L., Henning, W. G., \& Sasselov, D. D. 2010, AJ, 140, 1370

Kaltenegger, L., \& Sasselov, D. 2010, ApJ, 708, 1162

Kaltenegger, L., Sasselov, D., \& Rugheimer, S. 2013, ApJL, 775, L47

Kaltenegger, L., Segura, A., \& Mohanty, S. 2011, ApJ, 733, 35

Kaltenegger, L., \& Traub, W. A. 2009, ApJ, 698, 519

Kaltenegger, L., Traub, W. A., \& Jucks, K. W. 2007, AJ, 658, 598

Kane, S. R., Hill, M. L., Kasting, J. F., et al. 2016, ApJ, 830, 1

Kane, S. R., \& Hinkel, N. R. 2013, ApJ, 762, 7

Kasting, J. F., \& Ackerman, T. P. 1986, Sci, 234, 1383

Kasting, J. F., Kopparapu, R., Ramirez, R. M., \& Harman, C. E. 2014, PNAS, 111,12641

Kasting, J. F., Pollack, J. B., \& Crisp, D. 1984, JAtC, 1, 403

Kasting, J. F., Whitmire, D. P., \& Reynolds, R. T. 1993, Icar, 101, 108

Kopparapu, R. K., Ramirez, R., Kasting, J. F., et al. 2013, ApJL, 767, L8

Lissauer, J. J., Fabrycky, D. C., Ford, E. B., et al. 2011, Natur, 470, 53

Luque, R., Pallé, E., Kossakowski, D., et al. 2019, A\&A, 628, A39

Luyten, W. J. 1942, POMin, 2, 242

Manabe, S., \& Wetherald, R. T. 1967, J. Atmos. Sci., 24, 241

Morley, C. V., Kreidberg, L., Rustamkulov, Z., Robinson, T., \& Fortney, J. J. 2017, ApJ, 850, 121

Olson, S. L., Reinhard, C. T., \& Lyons, T. W. 2016, PNAS, 113, 11447

Pallé, E., Zapatero Osorio, M. R., Barrena, R., Montañés-Rodríguez, P., \& Martín, E. L. 2009, Natur, 459, 814

Ramirez, R. M., \& Kaltenegger, L. 2017, ApJL, 837, 1

Ramirez, et al. 2014, NatGe, 7, 59

Ranjan, S., Wordsworth, R., \& Sasselov, D. D. 2017, ApJ, 843, 110

Rimmer, P. B., Xu, J., Thompson, S. J., et al. 2018, SciA, 4, eaar3302

Rodler, F., \& Lopez-Morales, M. 2014, ApJ, 781, 54

Rugheimer, S., \& Kaltenegger, L. 2018, ApJ, 854, 19

Rugheimer, S., Kaltenegger, L., Segura, A., Linsky, J., \& Mohanty, S. 2015, ApJ, 809, 57

Rugheimer, S., Kaltenegger, L., Zsom, A., Segura, A., \& Sasselov, D. 2013, AsBio, 13, 251 
Schindler, T. L., \& Kasting, J. F. 2000, Icar, 145, 262

Schweitzer, A., Passegger, V. M., Cifuentes, C., et al. 2019, A\&A, 625, A68

Schwieterman, E. W., Kiang, N. Y., Parenteau, M. N., et al. 2018, AsBio, 18,663

Segura, A., Kasting, J. F., Meadows, V., et al. 2005, AsBio, 5, 706

Segura, A., Meadows, V. S., Kasting, J. F., Crisp, D., \& Cohen, M. 2007, A\&A, 472, 665

Segura, A., Walkowicz, L. M., Meadows, V., Kasting, J., \& Hawley, S. 2010, AsBio, 10, 751

Skrutskie, M. F., Cutri, R. M., Stiening, R., et al. 2006, AJ, 131, 1163

Snellen, I., de Kok, R., Le Poole, R., Brogi, M., \& Birkby, J. 2013, ApJ, 764, 182

Stassun, K. G., Oelkers, R. J., Pepper, J., et al. 2018, AJ, 156, 102

Toon, O. B., McKay, C. P., Ackerman, T. P., \& Santhanam, K. 1989, JGR, 94, 16287
Traub, W. A., \& Stier, M. T. 1976, ApOpt, 15, 364

Tuomi, M., \& Anglada-Escudé, G. 2013, A\&A, 556, A111

Udry, S., Bonfils, X., Delfosse, X., et al. 2007, A\&A, 469, L43

Udry, S., \& Santos, N. C. 2007, ARA\&A, 45, 397

Walker, J. C. 1977, Evolution of the Atmosphere (New York: Macmillan)

Walker, J. C. G., Hays, P. B., \& Kasting, J. F. 1981, JGR, 86, 9776

Walker, S. I., Bains, W., Cronin, L., et al. 2018, AsBio, 18, 779

Winn, J. N., \& Fabrycky, D. C. 2015, ARA\&A, 53, 409

Wordsworth, R. 2012, Icar, 219, 267

Wordsworth, R., Kalugina, Y., Lokshtanov, S., et al. 2017, GeoRL, 44, 665

Wordsworth, R., \& Pierrehumbert, R. 2013, Sci, 339, 64

Wordsworth, R., \& Pierrehumbert, R. 2014, ApJL, 785, L20

Zacharias, N., Finch, C. T., Girard, T. M., et al. 2013, AJ, 145, 44

Zeng, L., \& Sasselov, D. D. 2013, PASP, 125, 227

Zeng, L., Sasselov, D. D., \& Jacobsen, S. B. 2016, ApJ, 819, 127 